\title{
A sigma-delta analog to digital converter based on iterative algorithm
}

\author{
Mohammadmehdi Kafashan ${ }^{1 *}$, Mahboobeh Ghorbani ${ }^{2}$ and Farokh Marvasti ${ }^{1}$
}

\begin{abstract}
In this article, we present a new iterative algorithm aimed at improving the performance of the sigma-delta analog to digital (A/D) converter. We subject the existing sigma-delta modulator, without changing the configuration, to an iterative procedure to increase the signal-to-noise ratio of the reconstructed signal. In other words, we demonstrate that sigma-delta modulated signals can be decoded using the iterative algorithm. Simulation results confirm that the proposed method works very well, even when less complex filters are used. The simple and regular structure of this new A/D converter, not only makes realization of the hardware as ASIC or on FPGA boards easy, but also allows it to operate at high frequency levels with optimized power consumption and small chip area. Implementation of the design with an FPGA shows that experimental results are in agreement with the simulation results.
\end{abstract}

\section{Introduction}

Analog to digital (A/D) convertors are key in many applications of digital signal processing (DSP) and communication systems [1]. Conventional A/D converters consist of two steps: a sampling operation followed by a digital quantization. For uniform sampling, a common clock is operated in order to convert analog signals to digital values $[2,3]$. In a sigma-delta modulator (SDM), the original signal is highly oversampled and the internal clock operates at a much higher rate than the bandwidth of the signal; however, the oversampled signal is downsampled at the last stage of the A/D conversion. The noise introduced by signal distortion due to quantization degrades the $A / D$ performance [4-6].

Recovery of the original signal from its distorted version falls into the domain of the inverse problem. Although there are many solutions for this challenge, there isn't one direct analytic solution [7-9]. In this article, the iterative method is used to decrease the distortion caused by modulator. This innovative approach can be implemented without any changes to the structure of the SDM by adding a mathematical block after the demodulator of the SDM. Therefore, it can be used easily as a DSP chip for A/D conversion [10].

\footnotetext{
*Correspondence: mohammad.kafashan@gmail.com

1 Electrical Engineering Department and Advanced Communications Research Institute (ACRI), Sharif University of Technology, Tehran, Iran

Full list of author information is available at the end of the article
}

The rest of this article is organized as followed. The next section describes the iterative algorithm for recovery of bandlimited signals from their distorted versions. In section "Sigma-delta modulation using the iterative algorithm", the iterative solution for an A/D conversion is disclosed, followed by analysis and presentation of simulation results. Section "Implementation results of the iterative sigma-delta A/D converter" presents results of the new A/D converter implementation on an FPGA, and finally section "Conclusion" concludes this article.

\section{The iterative algorithm}

In this section, we first introduce the inverse problem and then explain how the iterative method can be used to solve this problem $[11,12]$. Consider the system shown in Figure 1, where the input to the system is unknown, but the output and the system (operator $G$ called distortion operator) itself are known. The relation between $y(t)$ and $x(t)$ is $y(t)=G\{x\}$. The goal is to find the signal $\widehat{x}(t)$ such that it becomes as identical as possible to the input signal $x(t)$.

A simple approach is to use the inverse system of $G$ which is noted as $G^{-1}$. Therefore, by feeding the output signal $y(t)$ into the reverse system, the input signal will be reconstructed with no error. However, it is usually difficult to obtain the inverse operator of $G$. Hence, we need a technique to approximate $G^{-1}$ based on $G$. In order to

\section{量 Springer}

(c) 2012 Kafashan et al.; licensee Springer. This is an Open Access article distributed under the terms of the Creative Commons Attribution License (http://creativecommons.org/licenses/by/2.0), which permits unrestricted use, distribution, and reproduction in any medium, provided the original work is properly cited. 


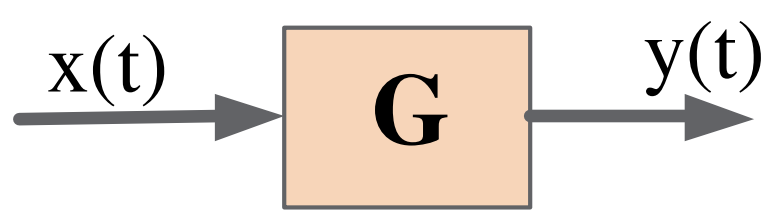

Figure 1 The known system with known output and unknown input signal.

overcome this problem, we unveil our iterative algorithm as follows:

$$
x^{(k)}(t)=x^{(k-1)}(t)+\lambda\left(G\{x(t)\}-G\left\{x^{(k-1)}(t)\right\}\right)
$$

where $x^{(k)}(t)$ is the $k$ th iteration and $G\{x(t)\}$ represents the output of the system when no iteration is used. Also, $\lambda$ denotes the relaxation parameter, which determines the convergence rate of the algorithm. This means that by using a larger value for $\lambda$, fewer iterations are needed to approximate the inverse of the system. In general, G can be either a linear or a non-linear operator. The discrete version of the iterative algorithm, when the distortion operator $\mathrm{G}$ is linear, can be written as:

$$
X^{(k)}=\widehat{G} X+(I-\widehat{G}) X^{(k-1)}
$$

where $I$ is an identity matrix of size $N \times N . X^{(k)}$ and $\widehat{G}=\lambda G$ in the above equation are $N \times 1$ and $N \times N$ matrix, respectively. By defining the operator $E=I-\widehat{G}$, the reconstructed signal after $\mathrm{k}$ iterations can be written as:

$$
X^{(k)}=\left(E^{k}+E^{k-1}+\cdots+E+I\right) X^{(0)}, \quad X^{(0)}=\widehat{G} X .
$$

The right hand side of the Equation (3) can be simplified using arithmetic progression such that:

$$
X^{(k)}=\left(\frac{I-E^{k+1}}{I-E}\right) X^{(0)}
$$

If the norm of the operator $E$ satisfies $\|E\|<1$, by increasing the number of iterations $k$, Equation (2) approaches the inverse system. Hence, $X^{(k)}$ converges to the original input signal $X$. The block diagram of the proposed iterative method is shown in Figure 2. In order to improve the performance of the approximated inverse system, it is only necessary to increase the number of blocks.

\section{Sigma-delta modulation using the iterative algorithm}

Sigma-delta modulator are well established to be used in high resolution A/D conversions [13,14]. The ratio between the sampling rate and twice the signal bandwidth (Nyquist rate) is defined as the oversampling ratio (OSR = $\left.f_{s} / f_{\text {Nyquist }}\right)$. The digital signal is digitally filtered to extract the analog signal information at high resolution. Typically the filters of the SDM concept are the critical elements and play an important role in determining the resolution of these A/D converters. Therefore, it is necessary to use filters which do not have complex architectures and can be readily utilized to achieve good performance in an A/D converter.

A block diagram of a first order SDM A/D converter is shown in Figure 3. The system consists of an analog SDM followed by a digital decimator. The modulator consists of an integrator, an internal $\mathrm{A} / \mathrm{D}$ converter or quantizer and a D/A converter (DAC) used in the feedback path. The process of noise shaping by the SDM can be viewed as pushing quantization noise power from the signal band to other frequencies. The modulator output is just a delayed version of the signal plus quantization noise that has been shaped. The modulator output can then be low-pass filtered to attenuate the out-of-band quantization noise and finally down-sampled to the Nyquist rate.

Some of the existing techniques to improve the performance of SDM A/D converters require increasing the OSR and using higher orders or multi-bits SDMs. The OSR can not exceeded a specific value, and it is limited by the frequency constraint of Sigma-Delta A/D converters, where $f_{\max }$ is the maximum frequency that can be supported. Higher order SDMs call for analog circuits which are more advanced and precise than those employed in the 1st order SDM. The main disadvantage of the multi-bit SDMs is that the multi-bit DAC cannot be easily fabricated using VLSI technology with adequate linearity. This is essential for high resolution conversion. In addition, the multi-bit output adds to the complexity of the digital low-pass filter (LPF) design that follows the modulator. Since, in multi-bit processing, the filter requires multi-bit hardware multipliers $[15,16]$.

The previous methods have mostly attempted to modify the architecture of SDM A/D converters in order to

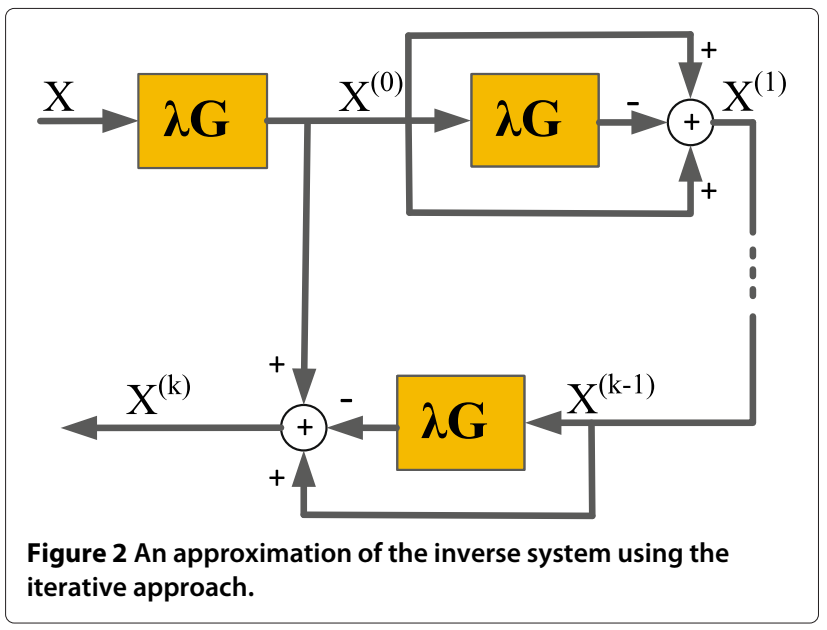



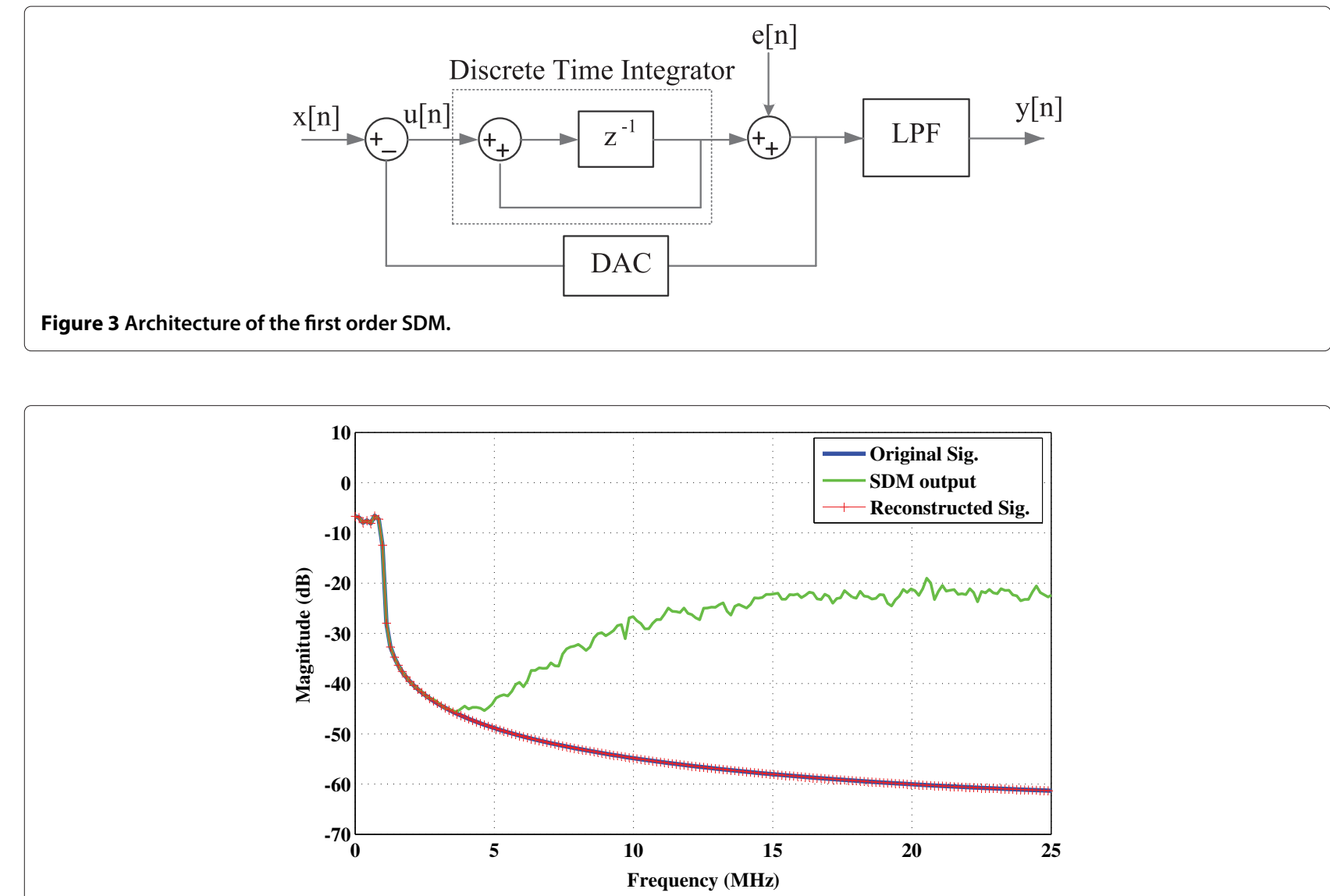

Figure 4 Spectra of the original signal (a bandlimited gaussian random signal) along with that of the recovered signals with and without using the iterative algorithm (SDM output is the output without iteration, and reconstructed signal is the output signal of the iterative procedure).
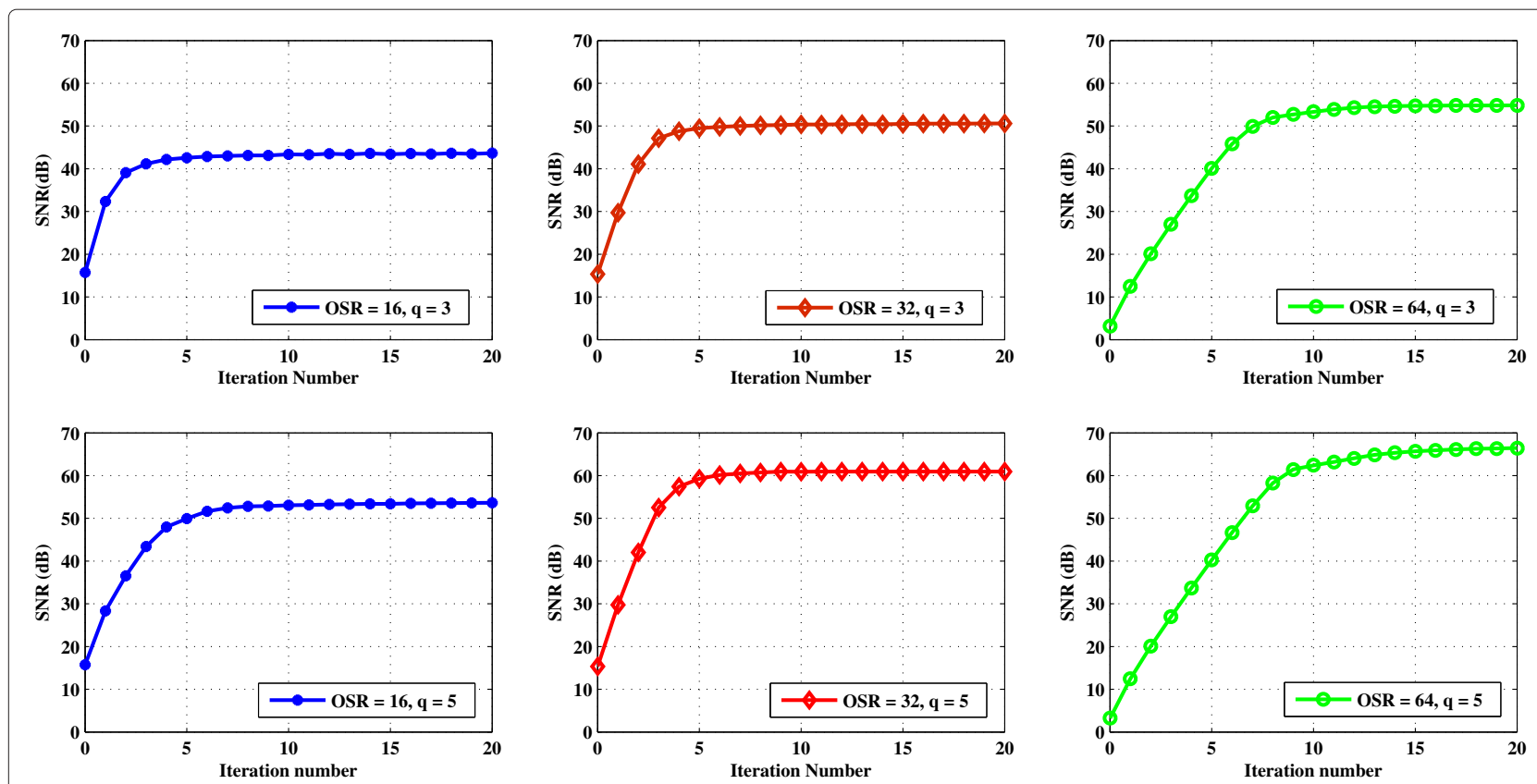

Figure 5 SNR of the reconstructed signal versus number of iterations for the first order SDM and FIR filter. 

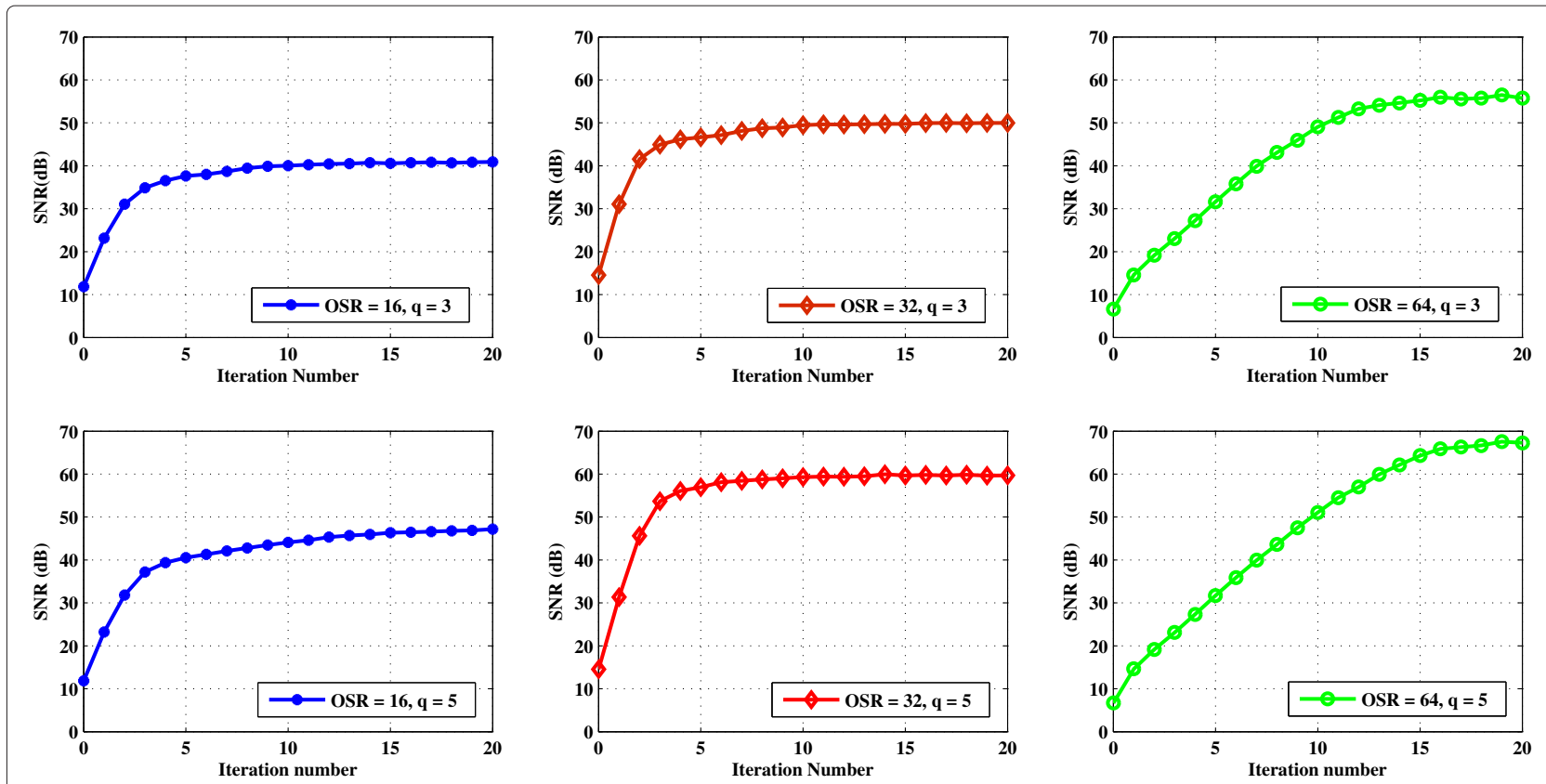

Figure 6 SNR of the reconstructed signal versus number of iterations for the first order SDM and IIR filter.

ensure improved A/D converter performance, while the proposed iterative approach exploits a mathematical signal processing algorithm to achieve the same goal. The distortion operator $G$ for SDM A/D conversion consists of a SDM and multistage LPF.

Simulations were run for various types of sigma-delta A/D converters for different OSRs, and it is demonstrated in Figures 4 and 5 that the iterative algorithm improves the performance of the first and second order SDM A/D converters. This algorithm can be used for improving the signal-to-noise ratio (SNR) of the reconstructed signal for all types of SDM A/D.

The spectrum of the original input signal (a bandlimited gaussian random signal), the SDM output and the reconstructed signal (using the iterative algorithm after five iterations) are depicted in Figure 4.

It can be seen from Figure 4 that the output of the SDM decreases the noise power in low frequencies which caused an increase in the apparent signal to noise ratio of the resultant signal. Hence, the spectrum of the original

Table 1 SNR of the reconstructed signal versus number of iterations for the second order SDM with $\lambda=1$

\begin{tabular}{|c|c|c|c|c|c|c|c|c|}
\hline \multirow[b]{2}{*}{ Filter type } & \multirow[b]{2}{*}{ OSR } & \multirow[b]{2}{*}{ Quantization bits } & \multicolumn{6}{|c|}{ Iteration number } \\
\hline & & & 0 & 2 & 4 & 10 & 15 & 20 \\
\hline \multirow[t]{6}{*}{ FIR } & \multirow[t]{2}{*}{16} & 3 & 18.15 & 43.58 & 55.10 & 57.06 & 57.19 & 57.15 \\
\hline & & 5 & 18.14 & 43.73 & 64.18 & 69.66 & 69.98 & 70.02 \\
\hline & \multirow{2}{*}{32} & 3 & 15.32 & 42.0 & 57.96 & 60.35 & 60.58 & 60.67 \\
\hline & & 5 & 15.32 & 42.16 & 65.08 & 77.51 & 78.33 & 78.48 \\
\hline & \multirow{2}{*}{64} & 3 & 21.79 & 29.63 & 44.67 & 67.02 & 66.46 & 67.07 \\
\hline & & 5 & 21.98 & 29.75 & 44.42 & 72.89 & 78.18 & 77.90 \\
\hline \multirow[t]{6}{*}{$\| \mathrm{R}$} & \multirow[t]{2}{*}{16} & 3 & 15.40 & 42.76 & 49.72 & 51.04 & 52.48 & 52.78 \\
\hline & & 5 & 15.40 & 43.66 & 61.61 & 63.07 & 63.40 & 64.12 \\
\hline & \multirow{2}{*}{32} & 3 & 16.12 & 45.24 & 57.24 & 60.16 & 60.79 & 61.26 \\
\hline & & 5 & 17.32 & 46.34 & 61.67 & 66.11 & 67.15 & 67.50 \\
\hline & \multirow{2}{*}{64} & 3 & 21.65 & 30.52 & 38.83 & 50.03 & 66.88 & 76.96 \\
\hline & & 5 & 21.65 & 30.52 & 38.83 & 50.07 & 67.32 & 81.53 \\
\hline
\end{tabular}




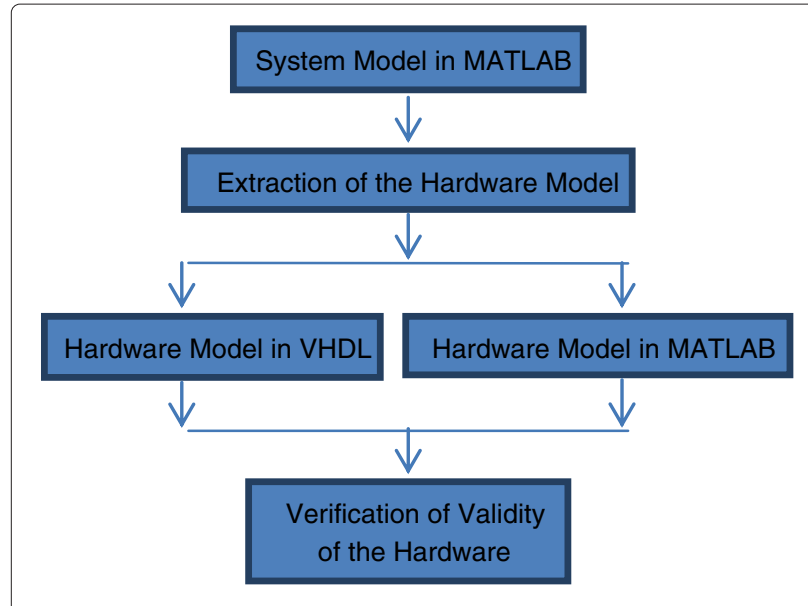

Figure 7 Design flow of the proposed A/D implementation.

signal and output of SDM have the same values at low frequencies. It is also clear that the frequency responses of the original and recovered signals are almost indistinguishable.

Impressive results have been obtained using the iterative algorithm. The SNR of the reconstructed signal versus number of iterations is displayed for the first and second order SDMs with the two numbers of quantization bits $(q=3,5)$. Two types of LPFs have been applied; the first is a simple FIR filter which has 90 taps with Hanning
Table 2 Design summary reported by Xilinx ISE for first order design of the proposed method

\begin{tabular}{llll}
\hline Logic utilization & Used & Available & $\begin{array}{l}\text { Utilization } \\
\text { (\%) }\end{array}$ \\
\hline Total number slice registers & 204 & 178,176 & 1 \\
Number used as flip flops & 199 & N.A. & N.A. \\
Number used as latches & 5 & N.A. & N.A. \\
Number of four input LUTs & 761 & 178,176 & 1 \\
Number of occupied slices & 459 & 89,088 & 1 \\
$\begin{array}{l}\text { Number of slices containing only } \\
\text { related logic }\end{array}$ & 459 & 459 & 100 \\
Number of slices containing & 0 & 459 & 0 \\
unrelated logic & & & 178,176 \\
$\begin{array}{l}\text { Total number of four input LUTs } \\
\text { Number used as logic }\end{array}$ & 764 & N.A. & N.A \\
Number used as a route-thru & 3 & N.A. & N.A. \\
Number of bonded IOBs & 143 & 960 & 14 \\
IOB flip flops & 5 & N.A. & N.A. \\
Number of BUFG/BUFGCTRLs & 1 & 32 & 3 \\
\hline
\end{tabular}

windowing (Figure 5) and the second one is the first type IIR filtering with the order of 9 (Figure 6). Also, three different OSRs are examined in our simulations. It can be concluded from the results that by increasing the value of the OSR, the quality of the reconstructed signal improves.

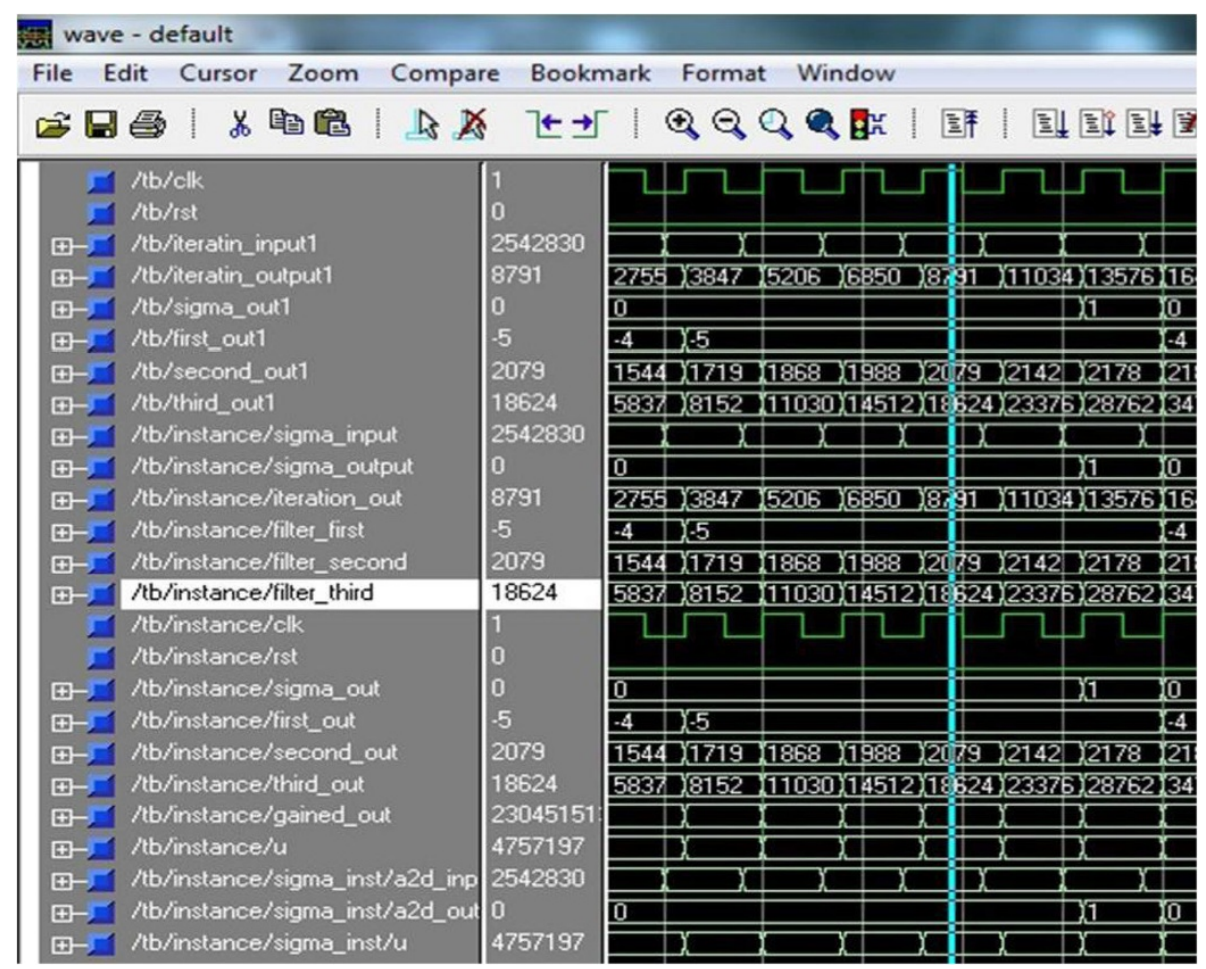

Figure 8 Waveform of the simulation of the design in Modelsim Software. 


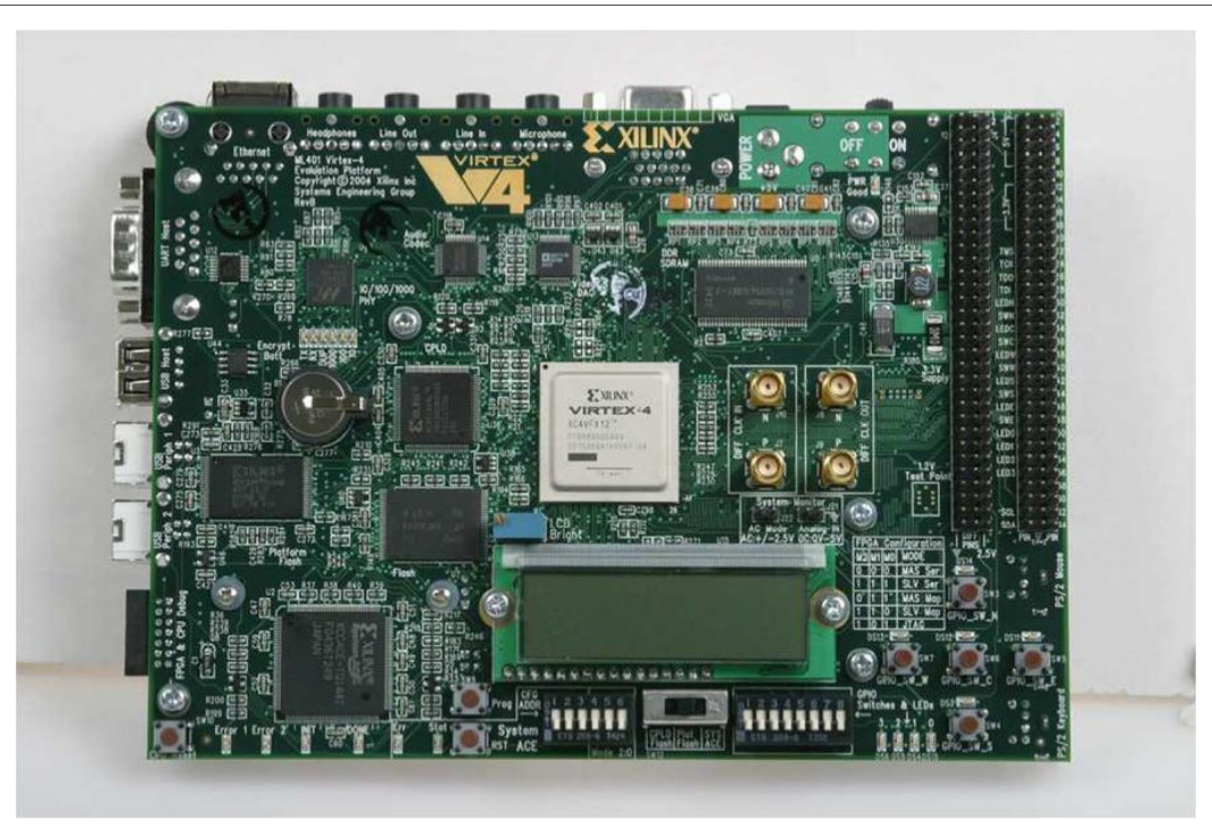

Figure 9 Xilinx ML403 evaluation board used for implementation the proposed algorithm.

Simulation results for the second order SDM with FIR and IIR LPFs are shown in Table 1. By comparing the results, we discover that the iterative algorithm enhances the performance of Sigma-Delta A/D converters for different types of LPFs and the numbers of quantization bits. Table 1 shows that the highest SNR value is about $60 \mathrm{~dB}$ for the second order IIR LPF which is obtained when OSR $=64$ and quantization bit is 5 .

\section{Implementation results of the iterative sigma-delta $A / D$ converter}

The sequence began by first implementing the proposed model in VHDL, then performing simulation runs and finally verifying the set up through the Mentor Graphic simulation software, Modelsim 6.0. A 32 bit fixed point number system was used for number presentation in order to better optimize system performance and power consumption. We also used synchronous flip flops as storing elements of the system variables and led feedback from the output of the sigma-delta.

From the original Matlab model of the design, we extracted the hardware model and in the meantime simultaneously generated an equivalent Matlab model for the hardware model of the design. Finally, after simulations the output of the hardware simulation of the model was compared with that of the Matlab model of the hardware implementation. In this way, we verified the correctness of the hardware model of the design. Figure 7 charts the design flow for this implementation.

We simulated the design in Modelsim Software using a 5,000 input sample array. Figure 8 shows a snapshot of the waveform of the simulation. We synthesized the design using Xilinx ISE for the first order SDMs. The device used was Xilinx Virtex4. The SNR values of the reconstructed signal were 23.88, 40.0595 and 40.6420 after one, two and three iterations, respectively. Looking at these figures, it is obvious that only two iterations are enough in order to achieve an increase of $15 \mathrm{~dB}$ in the SNR of the output signal. Table 2 shows the synthesize report generated by Xilinx ISE for the first order SDM.

Finally, we implemented the design on a real FPGA board (ML403 evaluation board shown in Figure 9). This required the inclusion of an on-board memory in the FPGA to store 5,000 input samples as an array, with the output of the design communicating to the PC through a Serial Cable and used RS232-UART to communicate with a PC. We then used Microsoft Windows Hyper Terminal Software to prompt get the generated output of the design to check in the Matlab Software.

The design was also evaluated in terms of power consumption. We synthesized the design using Synopsys Design Compiler (Version A-2007.12-SP5 for Linux) in

$\begin{aligned} & \text { Table } 3 \text { Power and area for design reported by synopsys } \\
& \text { design compiler }\end{aligned}$
\begin{tabular}{lcc}
\hline Cell Internal Power & $153.3550 \mu \mathrm{W}$ & $(58 \%)$ \\
Net Switching Power & $111.8264 \mu \mathrm{W}$ & $(42 \%)$ \\
Total Dynamic Power & $265.1814 \mu \mathrm{W}$ & $(100 \%)$ \\
Cell Leakage Power & $7.3691 \mu \mathrm{W}$ & \\
Total cell area & $2652.120016 \mathrm{~nm}^{2}$ \\
\hline
\end{tabular}


$65 \mathrm{~nm}$ technology and measured the power consumption by Synopsys Power Compiler Tool. Table 3 shows the power consumption of the iteration in terms of static and dynamic power. For dynamic power consumption, the results are presented for both internal and switching power.

\section{Conclusion}

This article presents a new iterative algorithm to convert analog signals to digital signals (A/D) by using a sigmadelta A/D converter. The iterative algorithm has proved itself as an effective tool to remove quantization noise caused by the quantizer and recover the original signal from its distorted version without changing the structure of the existing sigma-delta A/D converters. The main advantage of the proposed algorithm is that it improves the performance of these A/D converters and decreases the distortion caused by the SDM. This latter feature makes the application of lower degree filters possible, which leads to reduced cost and less complexity of the A/D converter design.

The sigma-delta A/D converters can find many practical applications as a result of their simple structures. Implementation of the iterative algorithm is easy, requiring no change to the configuration of the A/D converter in order to increase the number of iterations. Another notable advantage of the iterative algorithm is that it supports all types of SDMs with different orders and different numbers of quantization bits.

\section{Competing interests}

The authors declare that they have no competing interests.

\section{Author details}

${ }^{1}$ Electrical Engineering Department and Advanced Communications Research Institute (ACRI), Sharif University of Technology, Tehran, Iran. ${ }^{2}$ Computer

Engineering Department, Sharif University of Technology, Tehran, Iran.

Received: 2 September 2011 Accepted: 24 June 2012

Published: 18 July 2012

\section{References}

1. R Walden, Analog-to-digital converter survey and analysis. IEEE J. Sel. Areas Commun. 17(4), 539-550 (1999)

2. M Unser, Sampling-50 years after Shannon. Proc. IEEE. 88(4), 569-587 (2000)

3. J Candy, A Huynh, Double interpolation for digital-to-analog conversion. IEEE Trans. Commun. 34, 77-81 (1986)

4. R Schreier, G Temes, Understanding Delta-Sigma Data Converters (Wiley-IEEE Press, New York, 2004)

5. M Kozak, I Kale, Oversampled Delta-Sigma Modulators Analysis, Applications and Novel Topologies (Kluwer Academic, Boston, 2003)

6. M Kafashan, S Beygiharchegani, F Marvasti, Performance improvement of an optimal family of exponentially accurate sigma delta modulator. in Proc. IEEE International Conference on Signal Processing (ICSP), 2010, pp. 1-4

7. F Marvasti, Nonuniform Sampling: Theory and Practice (Kluwer Academic/plenum, New York, 2001)

8. K Grochenig, Acceleration of the frame algorithm. IEEE Trans. Signal Process. 41(12), 3331-3340 (1993)

9. M Kafashan, S Beygi, F Marvasti, Asynchronous analog-to-digital converter based on level-crossing sampling scheme. EURASIP J. Adv. Signal Process. 2011(1), 1-12 (2011)
10. A Oppenheim, R Schafer, J Buck, Discrete-Time Signal Processing. (Prentice Hall, Englewood Cliffs, 1999)

11. F Marvasti, M Analoui, M Gamshadzahi, Recovery of signals from nonuniform samples using iterative methods. IEEE Trans. Signal Process. 39(4), 872-878 (1991)

12. F Marvasti, P Clarkson, M Dokic, U Goenchanart, C Liu, Reconstruction of speech signals with lost samples. IEEE Trans. Signal Process. 40(12), 2897-2903 (1992)

13. B Razavi, Principles of Data Conversion System Design (IEEE Press, Piscataway, 1995)

14. F Marvasti, A/D Converters based on sigma delta modulators and iterative methods, United States Patent 7570186, 2009

15. S Norsworthy, R Schreier, G Temes, ICS Society, Delta-Sigma Data Converters: Theory, Design, and Simulation (IEEE Press, Piscataway, 1997)

16. R Gray, Quantization noise spectra. IEEE Trans. Inf. Theory. 36(6), 1220-1244 (1990)

doi:10.1186/1687-6180-2012-149

Cite this article as: Kafashan et al:: A sigma-delta analog to digital converter based on iterative algorithm. EURASIP Journal on Advances in Signal Processing 2012 2012:149.

\section{Submit your manuscript to a SpringerOpen ${ }^{\circ}$ journal and benefit from:}

- Convenient online submission

Rigorous peer review

- Immediate publication on acceptance

- Open access: articles freely available online

- High visibility within the field

- Retaining the copyright to your article

Submit your next manuscript at $>$ springeropen.com 